\title{
ON APPLYING VIRTUAL REALITY TO UNDERWATER ROBOT TELE-OPERATION AND PILOT TRAINING
}

\author{
Qingping Lin \\ Nanyang Technological University, Singapore \\ Chengi Kuo \\ University of Strathclyde, Glasgow, UK
}

\begin{abstract}
Virtual reality systems are being increasingly used for enhancing human perception of complex real-world or synthetic events. Serious applications outside entertainment range from education training to science and engineering. In this paper, we present our research work concerning the use of virtual reality in underwater robot tele-operation and training. We have proposed a novel concept of ROV safety domain to overcome the robot sensor error issue involved in virtual environment based underwater teleoperation approach. The detailed mathematical model of the ROV safety domain and its proof will be presented. In addition, we will examine how ROV safety domain can be used in underwater robot teleoperation, and present the architecture of our prototype virtual tele-operation system. Its current status and evaluation, as well as issues involved in its practical application will also be discussed.
\end{abstract}

\section{Introduction}

With the rapid advance in computer processing power and peripheral techniques, virtual reality offers the tools for enhancing human perception of complex events via simulating real-world or synthetic scenarios and object behaviors. Serious applications outside entertainment range from product design $[1,2]$ and simulation training $[3,4,5,6]$ to medical surgery [7] and aerospace industry[4,8].

One of the important VR attributes that facilitate these successful applications is the 3D graphical environment in which the users can freely navigate around and interact with virtual objects. This is especially useful for the applications that require real-time involvement with the task environment, e.g. robot tele-operation, since human tend to understand graphical information faster and easier than text and data. In robotic applications, virtual reality serves as a very valuable task visualization aids for planning and previewing robotic systems and tasks, for predicting robotic actions [9]. In addition, virtual environment allows the creation of an enriched learning environment including simulation of a wide range of operational environments, robot configurations, learning aids, and mission scenarios[10]. In fact, VR has provided useful enhancement to land-based and space robotic applications $[9,11]$.

In comparison with land-based and space robot operation, underwater robot tele-operation involves extra level of complexity due to visibility constraint, subsea positioning sensor calibration difficulty, and complicated underwater robot motion control[12]. Our research aims at developing a new approach for improving underwater robot tele-operation and pilot training. In this paper, we will begin with examining 
the challenges and issues facing the tele-operation of underwater robot before proposing virtual environment based approach for subsea robot tele-operation and pilot training. The design of our prototype virtual telepresence system will then be outlined, followed by robot sensor error issue involved in virtual environment based approach and the proposed solution. We will then present the current status and evaluation of the prototype system before discussing the issues involved in developing a practical virtual environment based system for assisting tele-operation of underwater robot. Finally, the key areas of further research will be outlined and the conclusions drawn

\section{Challenges of Underwater Robot Tele-operation and Pilot Training}

As offshore oil and gas exploration activities move to deeper and deeper water, underwater robots, e.g. remotely operated vehicles (ROVs), are gradually replacing divers for many of the subsea inspection, maintenance and repair tasks associated with offshore installations. Existing approach of ROV teleoperation relies on video camera image, sonar image, positioning equipment and physical model or memory of the offshore oil platform structure. The pilots have to take care to avoid umbilical entanglement while navigating the robot. The navigation task is hindered by visibility conditions, limited camera view field, and the complex nature of the structure. Muddy water can even make the telepresence system useless. As a result, even a very experienced pilot can easily get disoriented within the offshore structure due to the complexity of underwater navigation. The pilot's work is also very tedious and requires extremely high concentration[13]. Physical fatigue caused by long-time over-concentration increases the probability of collision or tether entanglement.

One improvement has been made on obtaining clearer camera image in underwater environment. Otsuka et. al[14] developed a ultra-high sensitive underwater color video camera that could produce much better underwater image than ordinary CCD camera. Zheng et. al[15] proposed a new laser 3D image display method, in which different frequency signal with distance information is used to display 3D image. Gordon et. al[16] reported their SM2000 Laser Line Scan System which could produce images in turbid water with a quality roughly 2-4 times that of available cameras by adopting new development in laser technology. There is no doubt that these approaches, to a certain extent, will improve the effectiveness of telepresence system. However, their performances are still visibility-dependent.

Associating synthetic background with live video image is another approach that has been introduced to improve the performance of telepresence system. Oderud et.al[17] proposed ROV teleoperation system that provides the operator with a clear view of subsea template by overlaying the realtime video coming from the camera mounted on the ROV onto an artificial background. However, the application is based on the condition that the ROV moves on fixed guiding rails on the subsea template. It is only suitable for guided robots which are designed to do few programmed operations on one particular worksite[18]. Hallset et. al[13] proposed another system to enhance visual presentation of the workplace with a wider and clearer view than a video camera can provide. This is done by matching sensor image with artificial image based on geometrical model of the workplace. However, this approach may fail due to poor underwater visibility that could lead to difficulty in matching the graphical representation with live video image. 
Acoustic approach has also been used to achieve better underwater imaging. Kamgar-Parsi et.al[19] and Blecher et. al[20] developed high-resolution acoustic imaging methods that can produce 3D image of underwater objects in great details. OmniTech AS[21] also developed an acoustic camera for applications in subsea survey and navigation. Acoustic approach has the advantage of not being affected by underwater visibility. However, this approach suffers from noise and multiple-path reflection effects. This limits its performance in ROV tele-operation inside complex offshore oil platform where multiple-path reflection, noise and robot motion effects become more serious.

For an ROV pilot to operate the robot efficiently, it is important that he always knows exactly the robot's spatial location and its surrounding environment. This requires a clear, steadily available 3D view of the scenario in which the robot is working.

On the other hand, pilots themselves have also played a very important role in efficient operation of the robot. It is the pilot that controls the motion of the robot, guides it from one location to another, and takes care its umbilical to avoid possible entanglement. The effective use of the robot is sensitive to the skills and training of the operator, both in terms of how fast work can be performed and in terms of safety[13,22]. Every operational decision of the pilot regulates the success of underwater intervention mission of the robot.

The challenging work of underwater navigation requires skillful and experienced pilot to guide the robot. Due to high cost of the robot, normally only experienced pilots are allowed to navigate the robot. Therefore it is difficult for a novice pilot candidate to gain ROV operation skill. These candidates have to go through certain training phases before they can start operating the real one.

Usually, ROV training simulator is based on simplified offshore structure configuration in deep tank. The trainees will benefit more from the training if the simulated scenario is realistic, or in other word, similar to the intended robot working environment. Unfortunately, the fact is that it is very difficult and extremely costly to simulate realistic ROV operation environment in the tank, not to mention their intended working situation. Therefore, existing training simulators only allow the trainees to be trained to get familiar with navigation instrument and general operational procedure. Furthermore, there is no literature record on training experienced pilots to get familiar with new robot working environment, i.e. an offshore structure that differs from the ones they previously worked with. Unfamiliar with the subsea environment causes hesitation or even making wrong operational decision, thereby results in costly timedelay or even worse collision/tether entanglement.

The review reveals that the main difficulty in achieving efficient tele-operation of ROV is the lack of clear, steadily available 3D view of the robot's working scenario and effective pilot training facility. Our research attempted to address the identified problems by using virtual environment based approach. Section 3 will present the system architecture of the proposed approach, robot sensor error issue involved in this approach and our solution. Current status and evaluation of the prototype system will be presented in Section 4 and 5 respectively. 


\section{Virtual Environment Based Approach for Underwater Robot Tele-operation and pilot Training}

Virtual environment enables reconstruction of real world events and scenarios in 3D graphics form based on geometrical models and object behaviors/sensor data. Thus with numerical position and orientation data of underwater robot, a 3D graphical representation of the robot and its surrounding environment can be generated. Single or multiple views of the structure and robot would overcome the limitation of cameras by showing the position of objects outside the camera's view field. This relieves the robot operator from imaging the robot's spatial location based on the sensor data. Furthermore, the graphical representation of underwater robot navigation scenario will be visibility-independent i.e. it can be generated continuously without being affected by visibility condition. Thus, 3D synthetic subsea scenario can be used for tele-operation of the robot if its position and orientation data is updated by underwater positioning system and orientation sensors. In addition, when robot location/orientation in the virtual environment is updated by a mathematical model of robot dynamics (e.g. robot motion generator controlled by joystick), the pilot can operate a simulated robot in the virtual subsea scenario without the risk of collision or tether entanglement. Underwater robot navigation scenario can be easily created when geometrical model of the oil platform is available. This could overcome the difficulty of providing realistic navigation scenario imposed by tank based training approach.

\subsection{SYSTEM ARCHITECTURE}

The aim of our research is to develop a system that can be used for both underwater robot teleoperation and pilot training. The basic idea is to couple robot sensor data with geometrical model of oil platform to create a virtual environment with sufficient accuracy for robot tele-operation, and to use simulated robot motion behavior for pilot training. The architecture of the system design is illustrated in Figure 1. There are two operation modes in the system: ROV tele-operation, and ROV pilot training. In ROV tele-operation mode, the robot's sonar sensor data will first be processed and matched with the geometrical model of the offshore platform to find out the robot's location and orientation[12]. The robot location/orientation is then used to index the platform's geometrical model to create a 3D virtual underwater navigation scenario. However, it should be noted that the synthetic scenario is generated based on geometrical model and sensor data. The synthetic scenario may not reflect the robot's real spatial location/orientation in relation to its surrounding environment. The errors existed in the robot's position and orientation sensor data will result in a 6 degree-of-freedom (DOF) offset in the robot's location rendered in the 3D synthetic environment. This could lead to the pilot making wrong operational decision, resulting in accidents. In the virtual telepresence system, we overcome this problem by displaying an ROV safety domain along the robot in the virtual environment. The safety domain is calculated based on sensor accuracy. ROV safety domain ensures that ROV is always inside the domain, thus enhancing the accuracy of the navigation information conveyed by the virtual environment. Sections $3.2-3.4$ give more details of the concept, its mathematical model and proof.

In simulation mode (i.e. ROV pilot training mode), the pilot will operate the robot as usual. The only difference from the tele-operation mode is that ROV motion is controlled by mathematical model of its dynamics. In this way the pilot can practice the ROV maneuvering process. Current effect and mission rehearsal are also incorporated into the system. This will allow the pilot to practice the planned mission in details. Thus hesitations caused by unfamiliar with the robot working scenario can be reduced, and subsea intervention work can be implemented more efficiently. 


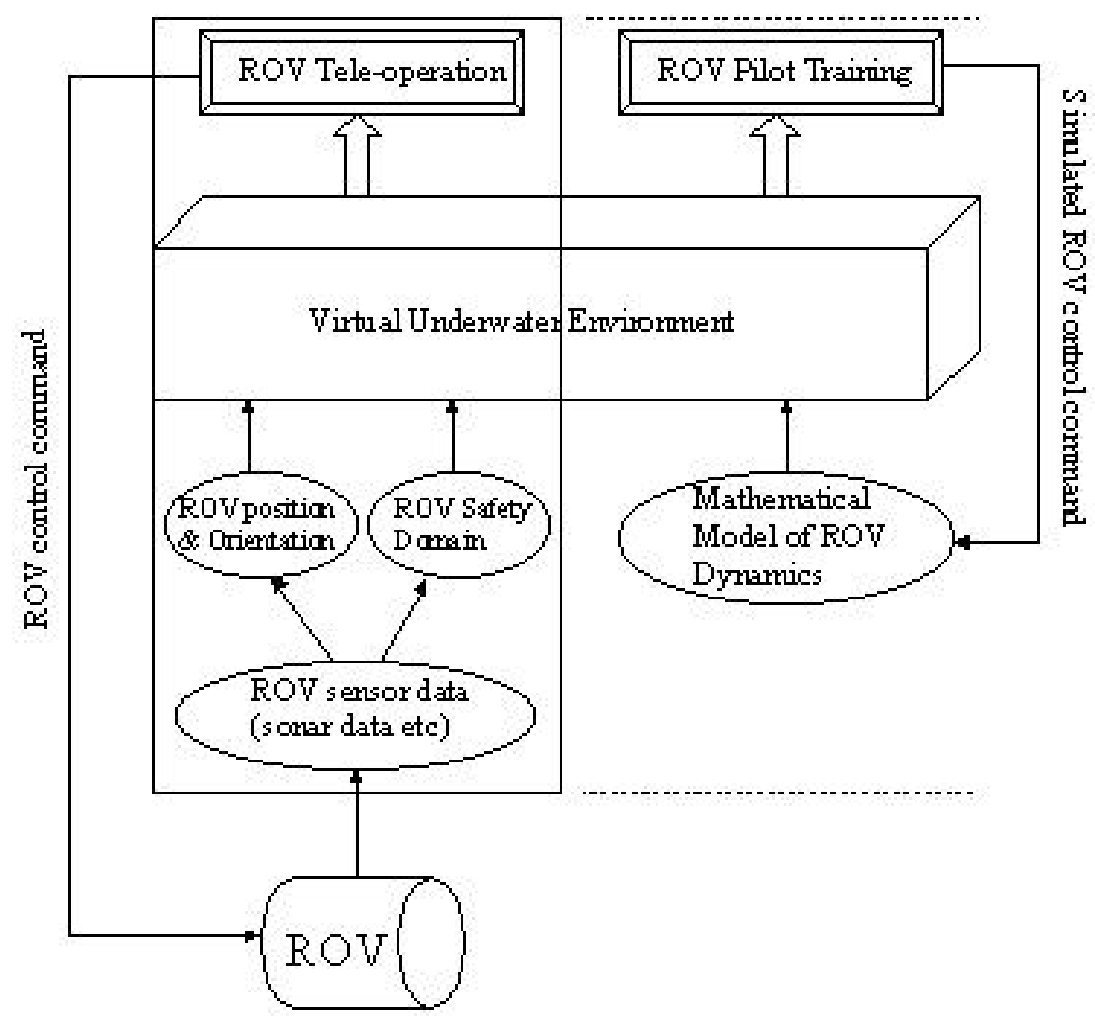

Figure 1: Architecture of the virtual environment based system for underwater robot tele-operation and pilot training.

\subsection{CREATION OF CORRECT VIRTUAL ENVIRONMENT}

The critical issue in using virtual environment for robot tele-operation is whether it can accurately mirror the robot's spatial location in relation to its surrounding environment in the real world. As discussed in Section 3.1, due to robot sensor errors, there will be a 6DOF robot location offset in the virtual environment. Existing methods of overcoming robot location offset are based on either calibrating the robot sensor errors or matching live video with synthetic graphics. Robot sensor error calibration has been actively researched in the robotic field. Related work include goal region method[23] and configuration space approach[24-29]. The goal region method is useful for locating moving object in a very confined 2D space, but not suitable for estimating spatial field of free-flying robot. Whereas configuration space approach is suitable for planar application and difficult to be implemented when 6 DOF robot sensor errors are involved, e.g. free-flying ROV. Other researchers have attempted to calibrate sensor errors in virtual environment using video image processing and matching techniques [11, 13, 30]. This approach is based on matching a graphically simulated virtual environment in 3D geometry and perspective with actual video camera views. This is useful when video image is clear, e.g. in land-based applications. However, in tele-operation of underwater robot, this condition may not always be satisfied due to poor visibility. To overcome the 6 DOF robot location offset in virtual environment, we propose the ROV Safety Domain (ROVSD) concept. 


\subsubsection{Definition of ROV Safety Domain}

ROV safety domain is defined as ROV's minimum spatial field which must be kept in order to prevent the potential danger of collision/tether entanglement caused by the errors in its position and orientation[8].

\subsubsection{Properties of ROV Safety Domain}

The purpose of introducing the concept of safety domain is to ensure that an ROV will not collide with an underwater structure if the domain is not in contact with the structure. Therefore, an ROV safety domain should have the following properties:

(1) None of ROV outline vertices will go beyond the safety domain if the orientation and position sensor errors are within the range of their own limits.

(2) The size of the domain must be the minimum one.

\subsubsection{Usage of ROV Safety Domain}

When creating a synthetic underwater scenario for assisting subsea navigation, the ROV itself and its surrounding objects (e.g. offshore structure) need to be included in the virtual environment. The spatial location of the vehicle in relation to its surrounding objects is determined by its positioning system. The possible errors in the vehicle positioning system result in uncertainty to the vehicle location in the virtual environment, i.e. there is a six degree-of-freedom image offset. And it is impossible to find the exact position and orientation of an ROV using existing navigation technology. In addition, the errors existed in position and orientation of an ROV are random. Therefore, it is impractical to eliminate the offset problem by directly adjusting the position of virtual telepresence images. This random robot location offset in the virtual environment makes it very difficult or even impossible for a pilot to navigate ROV safely inside an offshore structure.

ROV safety domain takes into account the vehicle dimension, position and orientation errors. If the true positioning sensor errors are within the maximum error ranges which are given by the accuracy of the underwater positioning system, then the ROV should always be inside the safety domain. As defined in the property of the ROV safety domain, the robot will not collide with offshore structure members if the domain is kept clear off them. Therefore, when the safety domain is displayed along with the robot in the virtual environment, the robot pilot will have the perception of the spatial field of ROV's location. Robot collision or tether entanglement can be avoided if the robot is treated as if it were enlarged to the size of its safety domain. With the virtual telepresence system, the ROV pilot no longer needs to imagine the robot's spatial location based on his interpretation of sonar image and often very unclear video image. He will be given a straightforward 3D visual information of the robot's location and its surrounding environment. As a result, with the aids of ROV safety domain and the virtual environment, the pilot can navigate the robot more efficiently and safely inside an offshore installation without being affected by underwater visibility and limited camera view field. A sample application of ROV safety domain for assisting subsea robot navigation in virtual environment is illustrated in Figure 6. 


\subsection{MATHEMATICAL MODEL OF ROV SAFETY DOMAIN}

The right hand coordinate system, illustrated in Figure 2, is built as follows: the origin of the coordinate system is placed at the geometrical center point of ROV; positive X-axis is pointing to ROV's head; positive Y-axis points to ROV's left-hand side while positive Z-axis points upwards. It is assumed that the orientation sensor accuracy is better than 20 degrees, and ratios of width to length, height to length and height to width are smaller than 2.5, which are reasonable for usable robot orientation sensors and normal ROV design. And ROV bounding box is used as its outline frame. Refer to section 3.4 for the rationale behind these assumed numbers. The robot positioning sensors use the fixed world coordinate system. Sensor errors will be transformed into local coordinate system during calculation of ROV safety domain. After the safety domain is computed, the coordinates of ROV safety domain will be transformed into the world coordinate system for display in the virtual environment.

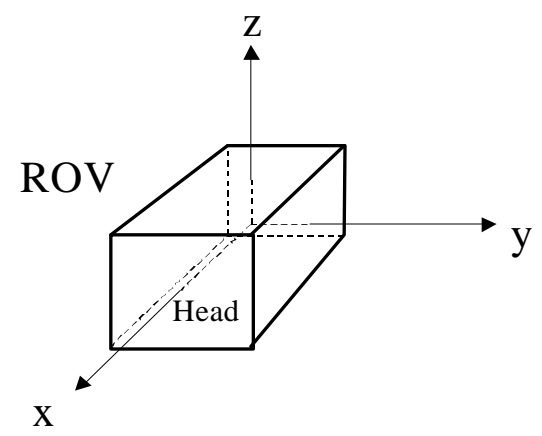

Figure 2: ROV coordinate system

Assume that ROV safety domain vertex $i$, with coordinate $\left(\mathrm{D}_{\mathrm{x}(\mathrm{i})}, \mathrm{D}_{\mathrm{y}(\mathrm{i})}, \mathrm{D}_{\mathrm{z}(\mathrm{i})}\right)$, corresponds to ROV vertex i, (x(i), y(i), z(i)). The calculation of coordinates of ROV safety domain vertices is as equation (1):

$$
\left[\begin{array}{llll}
D_{x(i)} & D_{y(i)} & D_{z(i)} & 1
\end{array}\right]=\left[\begin{array}{llll}
x(i) & y(i) & z(i) & 1
\end{array}\right] \cdot R_{D c}
$$

$\mathrm{R}_{\mathrm{Dc}}$ is ROV safety domain coefficient matrix. It is computed from equation (2):

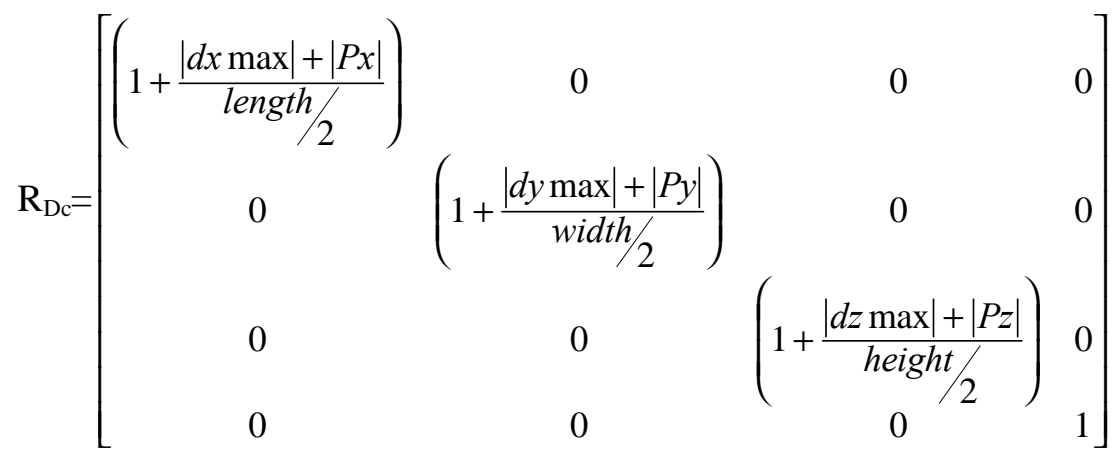

where $P x, P y, P z$ are x-, y-, z-components of ROV position error ranges; length, width, height are the dimension of ROV; The position error ranges include the positioning sensor error range and position offset error range. The positioning sensor error ranges are determined by the accuracy of the underwater positioning system. The position offset error ranges are the position errors caused the maximum time delay between a positioning fix and display of its location in the virtual environment. The time delay is 
determined by the refresh rate of the virtual environment system. $[|d x \max | \quad|d y \max | \mid d z \max ]$ are the maximum reach values of ROV outline vertices in $\mathrm{x}, \mathrm{y}, \mathrm{z}$ directions, caused by the error ranges in heading, roll, pitch. $[|d x \max ||d y \max ||d z \max |]$ can be derived from the equation (3):

$$
[|d x \max | \quad|d y \max | \quad|d z \max |]=\operatorname{Max}_{i=1}^{i=n} M_{j=1}^{j=15}\left[\left(|x(i, j)|-\frac{\text { length }}{2}\right)\left(|y(i, j)|-\frac{\text { width }}{2}\right)\left(|z(i, j)|-\frac{\text { height }}{2}\right)\right]
$$

where the function $\underset{i=1}{\stackrel{i=n}{a}}$ is defined as

$$
\underset{i=1}{\operatorname{Max}}\left[\mathrm{x}_{(\mathrm{i})} \mathrm{y}_{(\mathrm{i})} \mathrm{z}_{(\mathrm{i})}\right]=\left[\left(\underset{i=1}{\operatorname{Max}} \mathrm{x}_{(\mathrm{i})}\right)\left(\underset{i=n}{\operatorname{Max}} \mathrm{y}_{(\mathrm{i})}\right)\left(\underset{i=n}{\operatorname{Max}} \mathrm{z}_{(\mathrm{i})}\right)\right]
$$

$n$ is the total number of ROV vertices; $(x(i, j), y(i, j), z(i, j))$ is the new coordinate of the corresponding ROV vertex (x(i), y(i), z(i)) after performing the combined rotation which is indicated by $\mathrm{j}$. $\mathrm{j}$ represents the order of rotation with respect to pitch (rotation about Y-axis), roll ( rotation about X-axis), heading (rotation about Z-axis). $(x(i, j), y(i, j), \quad z(i, j))$ can be found from equation (5):

$$
[x(i, j) \quad y(i, j) \quad z(i, j) \quad 1]=\left[\begin{array}{llll}
x(i) & y(i) & z(i) & 1
\end{array}\right] \cdot \mathrm{R}_{(\mathrm{j})}
$$

$\mathrm{R}_{(\mathrm{j})},(\mathrm{j}=1,2,3, \ldots \ldots, 15)$ represents $\left(\mathrm{R}_{\mathrm{x}}\right),\left(\mathrm{R}_{\mathrm{y}}\right),\left(\mathrm{R}_{\mathrm{z}}\right),\left(\mathrm{R}_{\mathrm{x}} \cdot \mathrm{R}_{\mathrm{y}}\right),\left(\mathrm{R}_{\mathrm{x}} \cdot \mathrm{R}_{\mathrm{z}}\right),\left(\mathrm{R}_{\mathrm{y}} \cdot \mathrm{R}_{\mathrm{x}}\right),\left(\mathrm{R}_{\mathrm{y}} \cdot \mathrm{R}_{\mathrm{z}}\right),\left(\mathrm{R}_{\mathrm{z}} \cdot \mathrm{R}_{\mathrm{x}}\right)$, $\left(\mathrm{R}_{\mathrm{z}} \cdot \mathrm{R}_{\mathrm{y}}\right), \quad\left(\mathrm{R}_{\mathrm{x}} \cdot \mathrm{R}_{\mathrm{y}} \cdot \mathrm{R}_{\mathrm{z}}\right),\left(\mathrm{R}_{\mathrm{x}} \cdot \mathrm{R}_{\mathrm{z}} \cdot \mathrm{R}_{\mathrm{y}}\right),\left(\mathrm{R}_{\mathrm{y}} \cdot \mathrm{R}_{\mathrm{x}} \cdot \mathrm{R}_{\mathrm{z}}\right), \quad\left(\mathrm{R}_{\mathrm{y}} \cdot \mathrm{R}_{\mathrm{z}} \cdot \mathrm{R}_{\mathrm{x}}\right),\left(\mathrm{R}_{\mathrm{z}} \cdot \mathrm{R}_{\mathrm{x}} \cdot \mathrm{R}_{\mathrm{y}}\right)$ and $\left(\mathrm{R}_{\mathrm{z}} \cdot \mathrm{R}_{\mathrm{y}} \cdot \mathrm{R}_{\mathrm{x}}\right)$ respectively; $R_{x}$ is the transformation matrix of roll error range, $R_{y}$ is transformation matrix of pitch error range while $R_{z}$ is transformation matrix of heading(yaw) error range.

Matrices of $R_{x}, R_{y}$ and $R_{z}$ are $3 D$ coordinate rotation transformation matrix as follows:

$$
\begin{aligned}
& \mathrm{R}_{\mathrm{x}}=\left[\begin{array}{cccc}
1 & 0 & 0 & 0 \\
0 & \cos \varphi & \sin \varphi & 0 \\
0 & -\sin \varphi & \cos \varphi & 0 \\
0 & 0 & 0 & 1
\end{array}\right],(\varphi \text { is roll error range }) \\
& \mathrm{R}_{\mathrm{y}}=\left[\begin{array}{cccc}
\cos \theta & 0 & -\sin \theta & 0 \\
0 & 1 & 0 & 0 \\
\sin \theta & 0 & \cos \theta & 0 \\
0 & 0 & 0 & 1
\end{array}\right],(\theta \text { is pitch error range }) \\
& \mathrm{R}_{\mathrm{z}}=\left[\begin{array}{cccc}
\cos \omega & \sin \omega & 0 & 0 \\
-\sin \omega & \cos \omega & 0 & 0 \\
0 & 0 & 1 & 0 \\
0 & 0 & 0 & 1
\end{array}\right],(\omega \text { is yaw error range })
\end{aligned}
$$




\subsection{PROOF OF THE MATHEMATICAL MODEL OF ROV SAFETY DOMAIN}

Let us consider the y component first, from equation (3), the maximum y reach value will be

$$
|\operatorname{dymax}|=[\underset{i=1}{\max } \underset{j=1}{\operatorname{Max}}|\mathrm{y}(\mathrm{i}, \mathrm{j})|]-\text { width} / 2
$$

Substitute $|d y \max |$ value into equation (2), the y component of $\mathrm{R}_{\mathrm{Dc}}$ will be

$$
\left(\max _{i=1}^{n} \max _{j=1}^{15}|y(i, j)|+|P y|\right) /(\text { width / 2) }
$$

Solve equation (1) and (2), y component of vertex i of ROV safety domain $\mathrm{D}_{\mathrm{y}(\mathrm{i})}$ is as follows:

$$
\mathrm{D}_{\mathrm{y}(\mathrm{i})}=y(i)\left(\max _{i=1}^{n} \max _{j=1}^{15}|y(i, j)|+|P y|\right) /(\text { width / 2) }
$$

Now the true maximum y component of vertex $i$ is

$$
\mathrm{y}_{\max (\mathrm{i})}=[\underset{j=1}{15}|\mathrm{y}(\mathrm{i}, \mathrm{j})|]+|P y|
$$

Since $|\mathrm{y}(\mathrm{i}, \mathrm{j})| \leq \underset{i=1}{\operatorname{Max}} \mid \mathrm{y}(\mathrm{i}, \mathrm{j})$, it follows that $[\underset{j=1}{\operatorname{Max}}|\mathrm{y}(\mathrm{i}, \mathrm{j})|] \leq[\underset{i=1}{\operatorname{Max}} \underset{j=1}{\operatorname{Max}}|\mathrm{y}(\mathrm{i}, \mathrm{j})|]$.

It is assumed that bounding-box is used as the outline frame of the ROV. With the assumption, $y(i) /\left[\right.$ width/2]=1will be valid. Thus $y_{\max (i)} \leq D_{y(i)}$. This assures that the true maximum y component of vertex $\mathrm{i}$ will not be larger than its corresponding $\mathrm{y}$ value in ROV safety domain. $\mathrm{x}$ and $\mathrm{z}$ components can be proved in the similar way. The above has proved that the algorithm will always generate an ROV safety domain that contains the original ROV envelope.

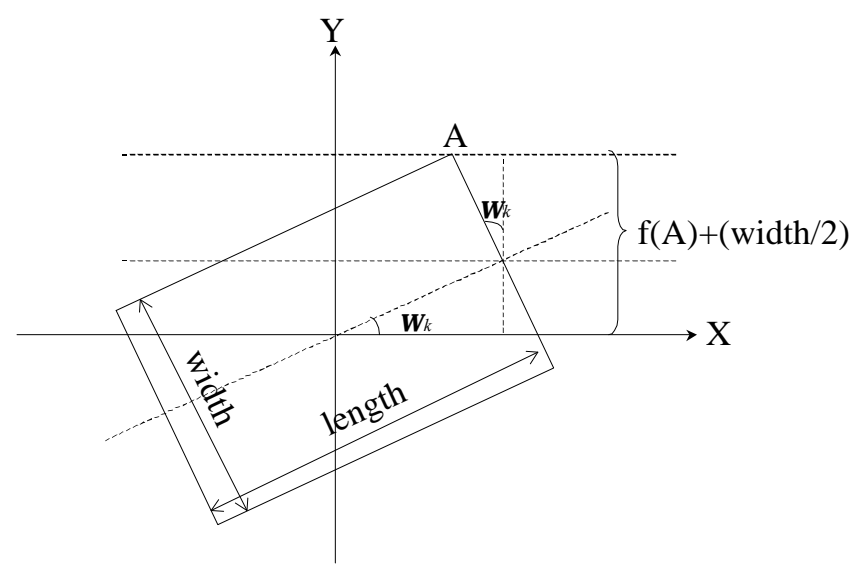

Figure 3: $2 D$ view of ROV bounding box vertex's offset caused by yaw sensor error $\omega_{k}$. 
The following paragraphs prove that for given sensor error ranges, the size of the ROV safety domain, generated by the algorithm, will be minimal. Now let's assume that $f(A)$ is the function representing the $y$ reach value of the vertex A of ROV bounding box when there is a yaw error $\omega_{k}$, as shown in Figure 3, then $\mathrm{f}(\mathrm{A})=$ length $* \sin \left(\omega_{k}\right) / 2+$ width $^{*} \cos \left(\omega_{k}\right) / 2$-width $/ 2$. The first order derivetive of $\mathrm{f}(\mathrm{A})$ will be $\mathrm{f}^{\prime}(\mathrm{A})=$ length $* \cos \left(\omega_{k}\right) / 2$-width* $\sin \left(\omega_{k}\right) / 2$.

With the assumptions, $\left|\omega_{k}\right| \subset[0,20]$ and (width/length) $\leq 2.5$ given in Section 3.3 , f '(A) will be $\geq 0$. This indicates that $\mathrm{f}(\mathrm{A})$ is a increasmental function under the condition of the asumptions. Thus for any given $\left|\omega_{k}\right| \subset[0, \omega],\left|\left[\left.\mathrm{f}(\mathrm{A})\right|_{\omega k}\right]\right| \leq\left|\left[\left.\mathrm{f}(\mathrm{A})\right|_{\omega}\right]\right|$ is valid. The equal condition will only be satisfied when $\left|\omega_{k}\right|=\omega$. And $\left|\left[\left.\mathrm{f}(\mathrm{A})\right|_{\omega}\right]\right|$ is, in fact, the value of $|d y \max |$. Now for any given $\left|P y_{\mathrm{k}}\right| \subset[0,|P y|]$, the true offset value of the ROV bounding box vertex $\mathrm{A}$ will be $\left(\left|\left[\left.\mathrm{f}(\mathrm{A})\right|_{\omega k}\right]\right|+P y_{\mathrm{k}}\right) \leq \quad(|d y \max |+|P y|) . \quad\left(\left|\left[\left.\mathrm{f}(\mathrm{A})\right|_{\omega k}\right]\right|+P y_{\mathrm{k}}\right)=$ $(|d y \max |+|P y|)$ will be valid only when both $\left|\omega_{k}\right|=\omega$, and $\left|P y_{\mathrm{k}}\right|=|P y|$ are sastified.

Similar results can be achieved for $\mathrm{x}$ and $\mathrm{z}$ component. This proves that as long as ROV sensor errors are within their own error ranges, the resulting maximum reach value of ROV outline vertex will be no larger than that caused by the sensor' boundary errors, that is, the size of the ROV safety domain is the minimum one.

A simulation software was also written to test whether the mathematical model of ROV safety domain satisfies its definition and properties. This software allows both visual and numerical assessment of the mathematical model using computer randomly generated robot's position and orientation errors. And the outline dimension of ROV SUPER SCOPPIO which has the typical shape of working class ROVs is used in the test. The test results show that ROV outline vertices may have the chance to reach the boundary of the domain but never exceed it. This satisfies the requirement of the definition. Sample figures representing numerical test results and the graphical assessment are illustrated in Figure 4 and Figure 5. Figure 4 shows the test results of X-boundary of the safety domain. Y-and Z-boundary tests have similar results, see reference[31] for details.

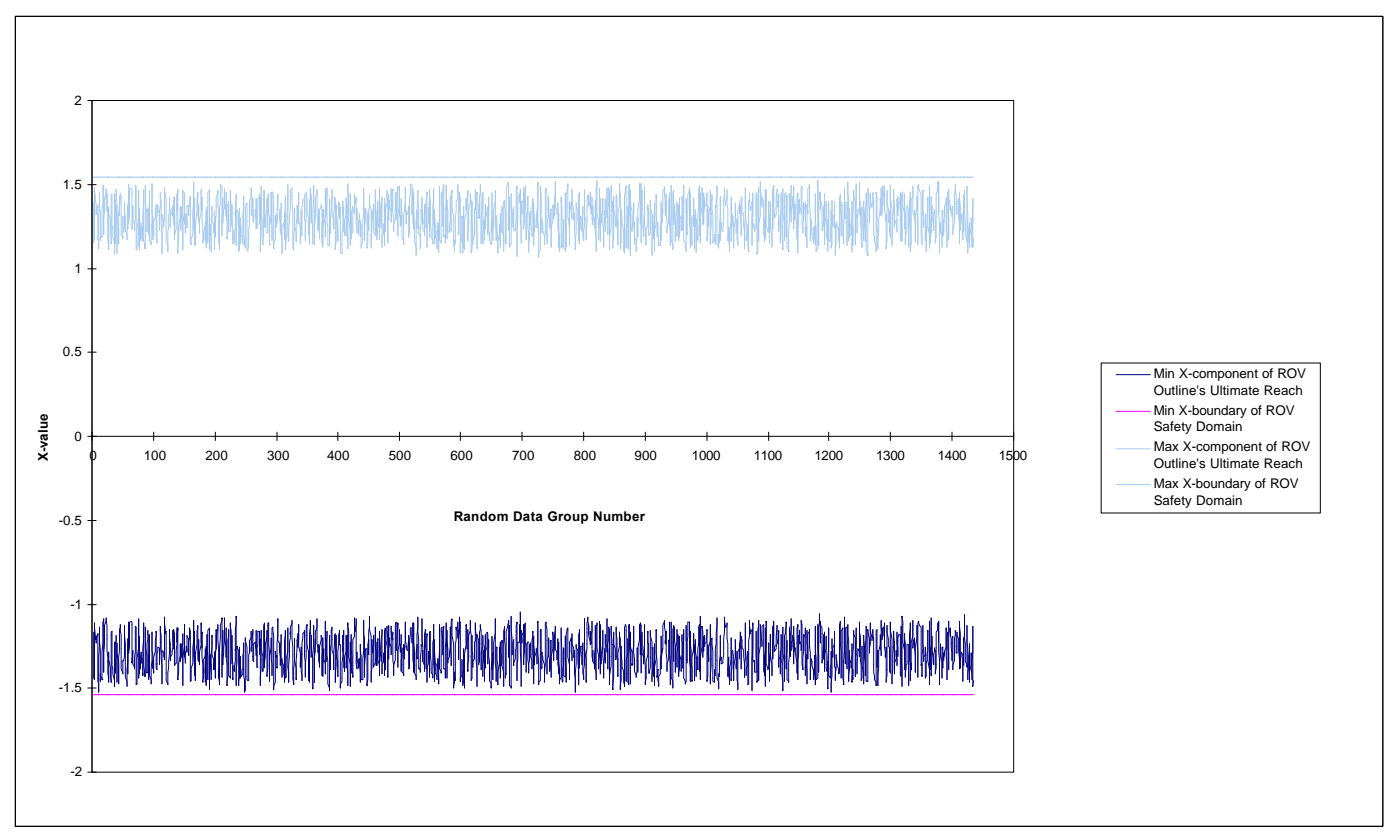

Figure 4: X-boundary test results of ROV safety domain 


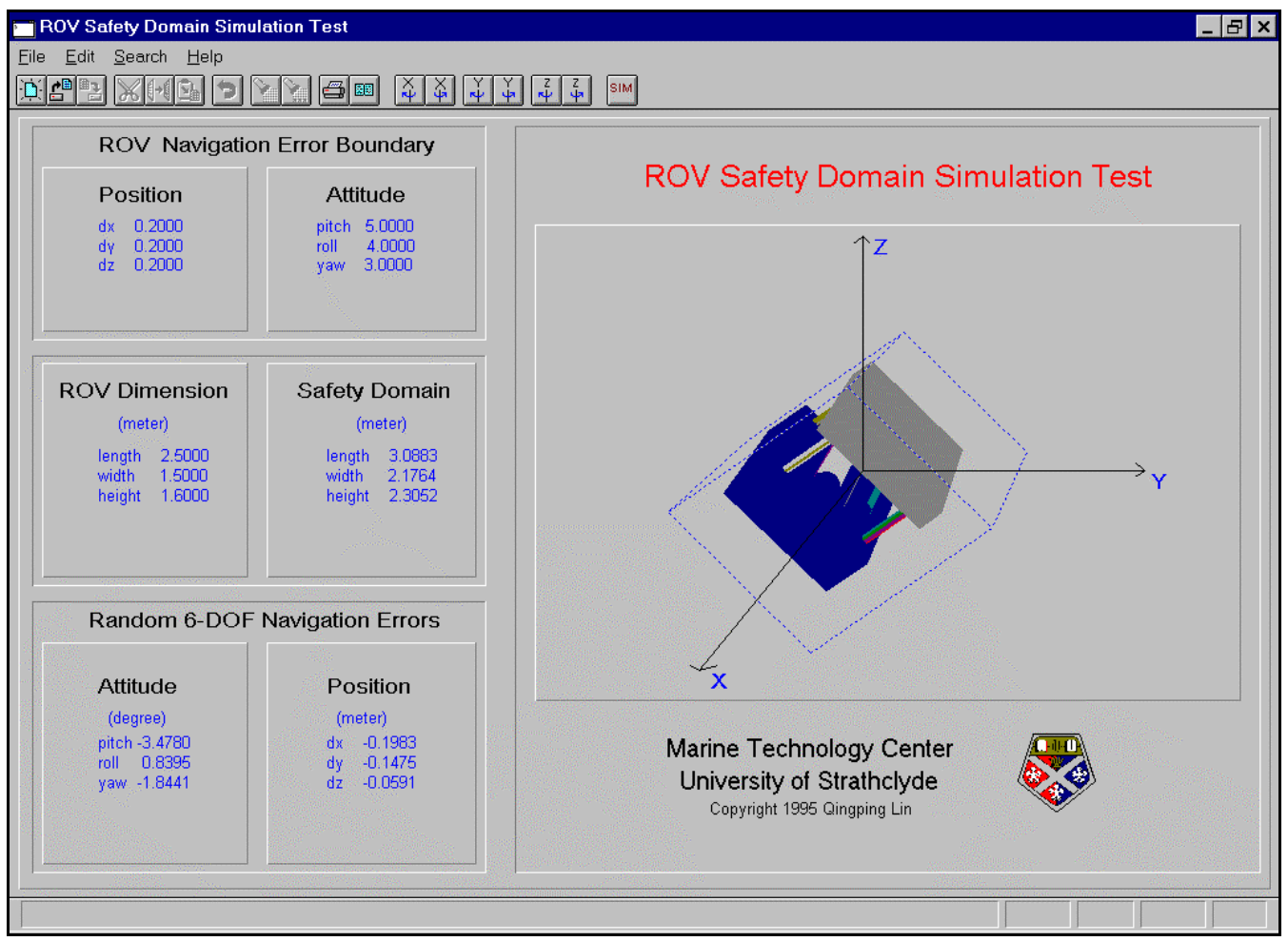

Figure 5: ROV safety domain simulation test: a sample view of graphical assessment display.

\section{Current Status}

A prototype virtual telepresence system for assisting ROV tele-operation has been developed, as shown in Figure 6-8. The system takes robot's position/ orientation data from a sonar based underwater positioning unit[12]. The positioning unit finds the robot's location by matching sonar image with 3D geometrical model of the offshore installation. The sonar positioning software has been developed and tested in both lab and offshore environment. Evaluation of the sonar positioning software will be given in section 5. The virtual telepresence system will then generate synthetic subsea navigation scenario based on the robot location found by the sonar positioning unit, and geometrical models of the offshore structure[31-33].

At the same time, the system will calculate the ROVSD on-line based on the robot sensor accuracy and positioning confidence. The calculation result of ROVSD will be displayed in the virtual environment, as shown in Figure 4, in the form of a white box wrapping the robot. It tells the pilot that ROV is inside this box. So long as the ROVSD is clear off the structure members, the robot will be free from collision. With the aid of these facilities, the ROV pilot no longer needs to imagine the robot's spatial location based on his interpretation of sonar data and video image, which is often very unclear. He will be given a straightforward 3D visual information of the robot's location and its surrounding environment. In addition, the display of the robot's history path indicates the possible location of its tether, illustrated in Figure 6, tether entanglement can be avoided by flying the robot back along the history path. 


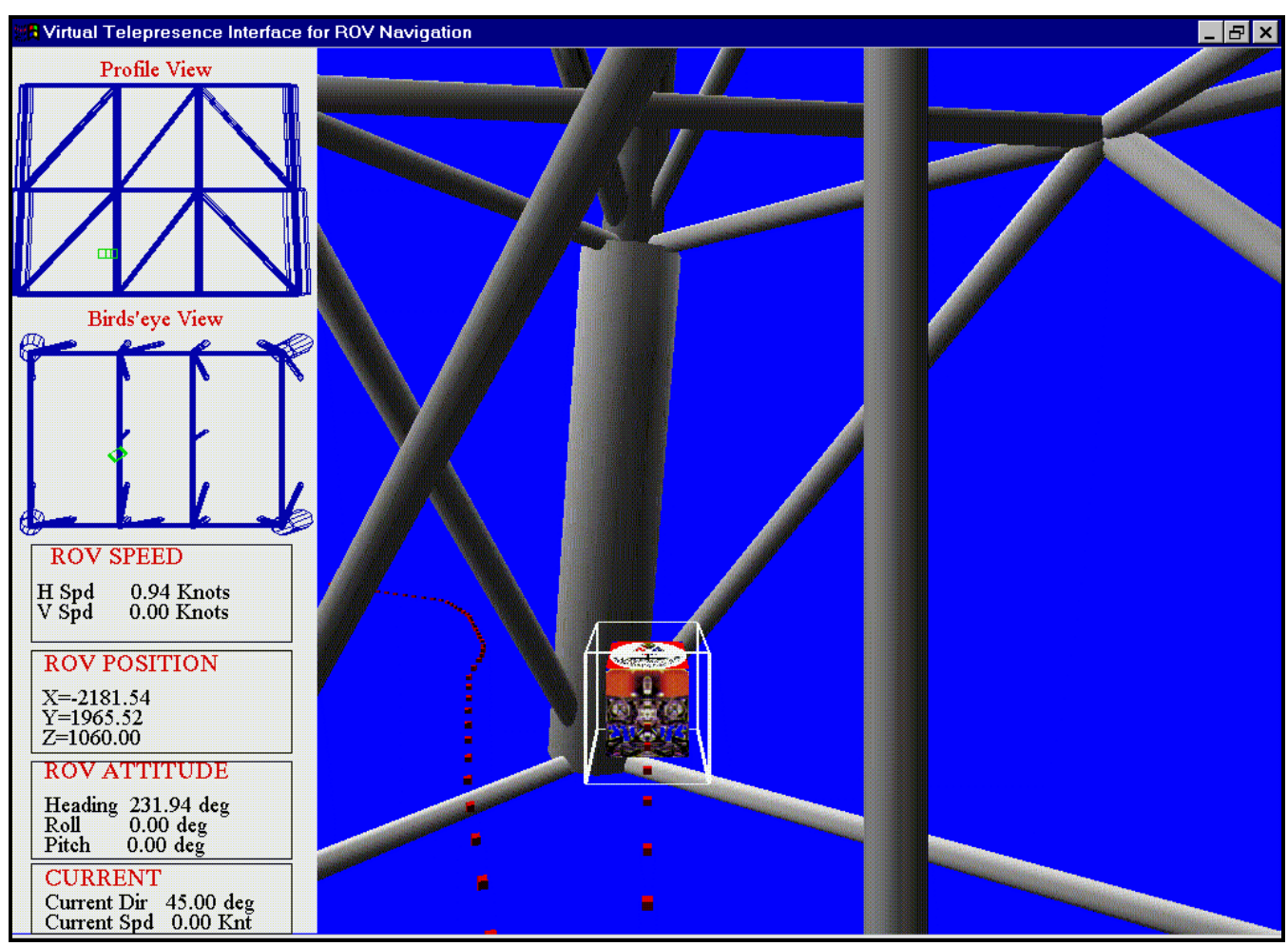

Figure 6: Sample screen capture of the virtual telepresence system, with camera view, profile view, bird's eye view, and history path display. The safety domain is displayed as a white box wrapping the robot.

Besides the function for assisting tele-operation of ROV, the system also provides pilot training and mission rehearsal features. In the simulation training mode, 3D geometrical model of any offshore installation can be loaded into the system to generate the virtual underwater navigation environment. The mathematical model of the robot dynamics is used to control the behavior of the robot. At present only a simplified mathematical model is used [31]. A novice ROV pilot can be trained to learn the process of the robot operation. Furthermore, either experienced or novice pilots can also be trained to get familiar with a new/intended robot working environment (e.g. offshore installations). This is important when a very vital part of the installation (e.g. well head) needs to be inspected[34]. In that case, the mission can be practiced before execution. The collision detection capacity of the system, as shown in Figure 7, will facilitate the assessment of the process of mission practice and training performance.

ROV's subsea intervention mission can be planned using the virtual telepresence system. With 3D geometrical model of an offshore structure, a virtual environment of the ROV's worksite can be built and stored as data base for subsea intervention work. The underwater mission can be planned in details. Thus the ROV pilot can practice the deployment procedure of the robot according the planned mission. This is especially important when a mission is complex and difficult or takes place in an important part of the offshore installation[34,35]. 


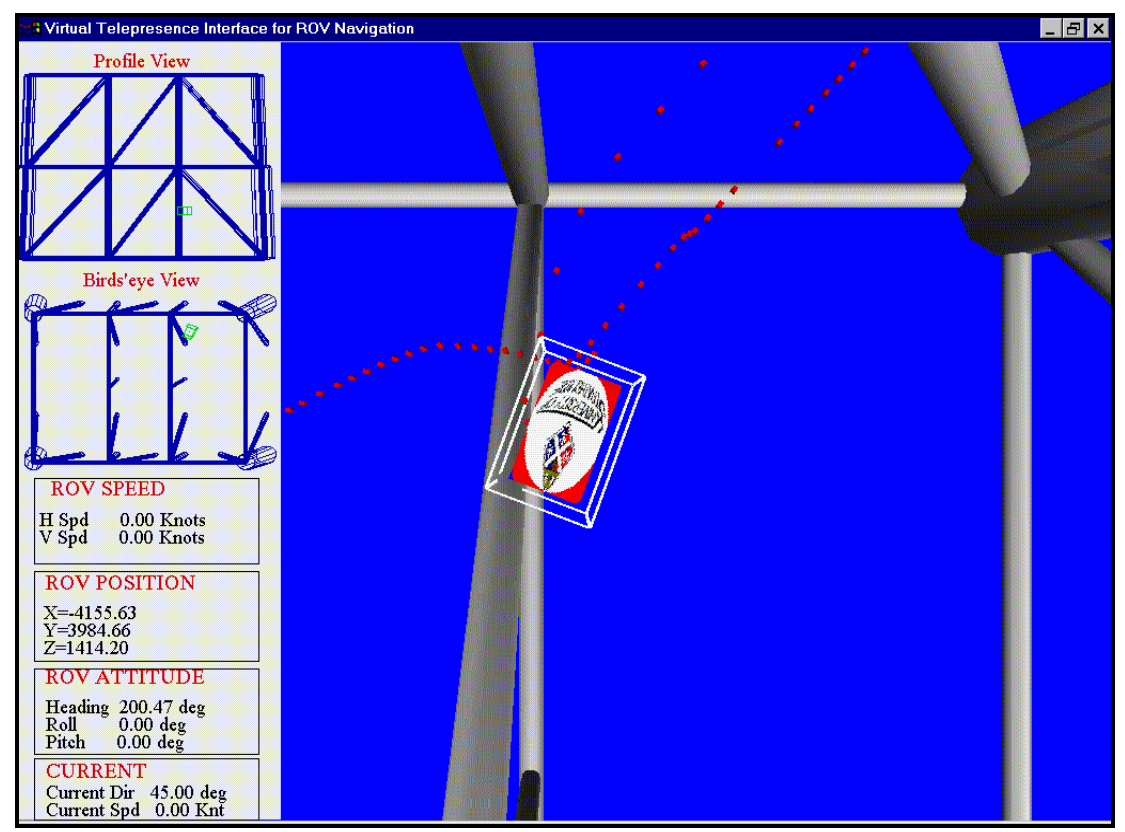

Figure 7: Screen capture of a collision detected by the virtual telepresence system during the simulation test of ROV guidance within Beryl Bravo platform.

During execution of the mission, the planned path can be displayed on the virtual worksite. This allows the pilots to simply follow the path. If precise robot positioning and control systems are linked to mission module, the ROV can follow the path accurately. Once the ROV is carefully moving along its course, the pilot can concentrate on the graphics display. This save the pilots from making real-time operational decision. Thus, the navigation and telepresence operation task is reduced to monitoring one graphics display. An example of displaying planned path on the virtual worksite is illustrated on Figure 8.

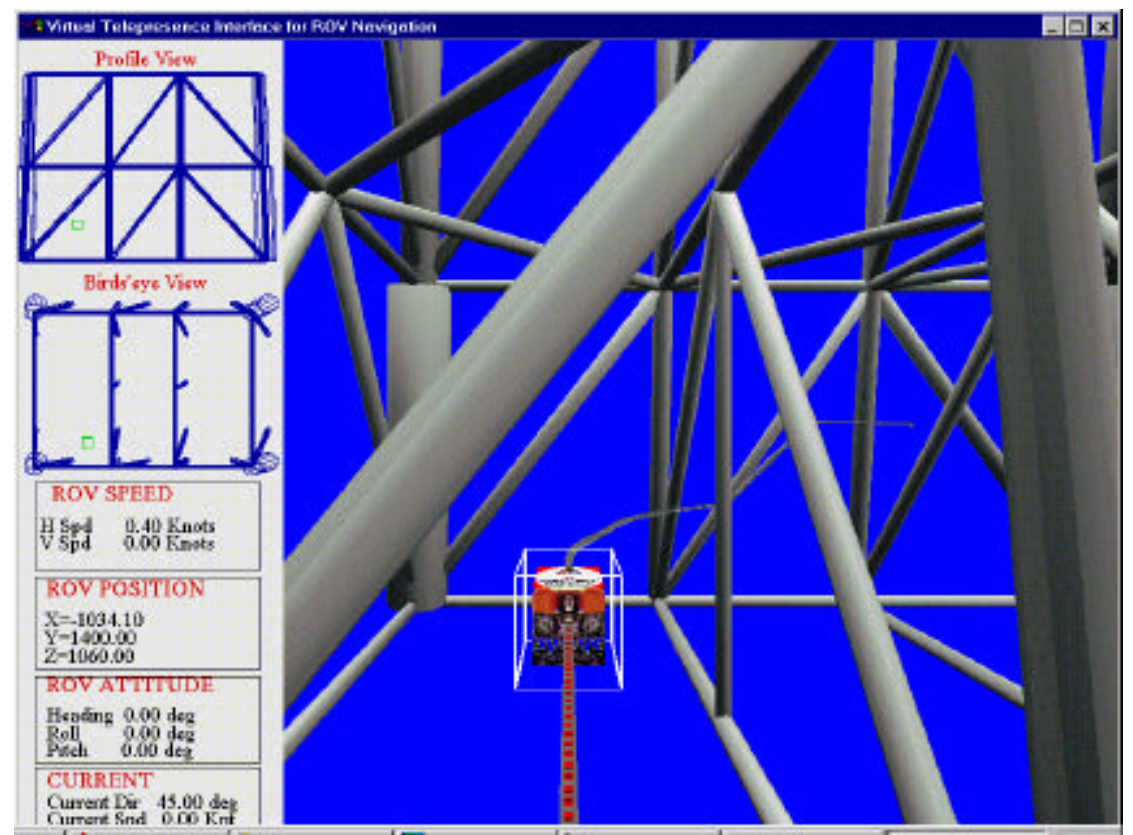

Figure 8: Display of mission path in the synthetic worksite within Beryl Bravo platform. 


\section{Experimental Evaluations}

Sonar data matching software has been tested in both lab and offshore environment. The tests have confirmed that by mapping sonar image with geometrical model of the offshore oil platform, the robot's spatial location can be found[12,36,37]. The positioning accuracy is $20 \mathrm{~cm}$. This is sufficient for ROV navigation. However, the positioning software is based on mechanical scanning sonar, which has high image quality but low refresh rate at about 5 seconds each frame. Although the image quality enhances the confidence of the sonar matching, the update rate is not sufficient for real-time navigation. However, the test results have proven the principle and algorithms used in the sonar matching[12, 36,37]. We are now working on using electronic scanning sonar that has high update rate, about $10 \mathrm{~Hz}$, but low image quality. This update rate should be tolerable for real-time ROV navigation as the robot is normally moving at slow speed within the offshore platform.

A series of tests on the prototype virtual telepresence system were carried out using geometrical model of Beryl Bravo platform and ROV SUPER SCOPPIO. In the experimental test, the robot's position and orientation was controlled by a joystick. The robot could be guided to any possible location inside an offshore structure in which subsea intervention work (e.g. maintenance, inspection etc.) could take place. The simulation test includes free flying robot within the offshore installation, current effect, ROV safety domain data processing and its graphics display, robot history path recording and display, and collision detection.

During the process of implementing ROV's subsea mission, tele-operation of the robot is assisted by the three views at the same time as shown in Figure 4. One is the primary view (camera view) in which the viewpoint is set to a position behind the robot with viewing orientation looking towards it so that it is always located within the range of the view field. The other two views are the bird's eye view, which helps the pilot to judge the horizontal location of the robot, and the profile view for identifying the robot's vertical location. In addition, the pilot can change the viewpoint and viewing orientation at any time. With these views, the ROV pilot will have a full perception of the robot's spatial location and its surrounding environment. Thus, the pilot can concentrate on maneuvering of the robot. This will relief the fatigue of pilot's work, which will ultimately increase the mission success probability. The system's impact on improving the efficiency of tele-operation of ROV was appreciated by the offshore operators during the simulation demonstration. The operators felt getting better orientation with the simultaneous display of multiple viewpoints and the robot's history track. Another finding is that the history track display will be not only useful in locating the tether location but also important for pilot shift changing. In existing approach, due to the difficulties in explicitly explaining tether location and robot position/ orientation as a result of similarity in structure members, the pilot normally has to exit the robot from the offshore platform before the next pilot can take over the duty. Display of robot history track in virtual environment will overcome this problem. The practical feasibility of the virtual telepresence system will, however, require an offshore trial to prove it.

The demo of the pilot training and mission rehearsal features had received some encouraging comments from the offshore operators. They felt that the pilots would be able to practice the robot operation procedures and acquire necessary subsea robot tele-operation skills without risking the physical robots. This would reduce the training cost and shorten novice pilot's learning cycle. It was also noted that safety of underwater mission would be improved as plans may be checked thoroughly using simulation and executed automatically under supervision of the ROV pilot, reducing the risk of human errors. However, the offshore operators felt that ROV hydrodynamics should be incorporated into the existing simplified mathematical model of ROV motion in order to generate realistic ROV behaviors in the virtual environment. This issue will be included in our future research work. 


\section{Discussions}

To accommodate the development of practical virtual environment based system for assisting teleoperation of ROV, there are a number of issues worth discussion. First of all, the system performance issue. The meaningfulness of the visual navigation information provided by the virtual telepresence system is regulated by its image accuracy and update rate. Indeed, the errors in robot's position and orientation can be overcome by the use of ROV safety domain. However, the accuracy of the 3D geometrical model of the offshore installation will also affect the correctness of the virtual navigation scenario. Therefore, accurate 3D geometrical models of the offshore installation should be used in order to make the visual information useful in practice. The 3D models, however, may not always be available due to the fact that normal engineering drawings are created in 2D format. In this case, it is necessary to convert the 2D drawings into 3D models. And the accuracy of 3D models should be kept at centimeter level. In addition, the 3D models should be built based on real construction models and any known changes (e.g. rearrangement of a structure member) should be updated. Even though the 3D model database is fully updated, there may be some unexpected objects (e.g. damaged/corroded structure member hanging around) in the real world environment inside an offshore platform. These objects must also be presented in the virtual environment in order to navigate the robot safely. This could be achieved by the use of acoustic imaging techniques[20,21].

On the other hand, update rate of the system graphical interface depends on the size of the offshore installation and graphics processing power of the computer used. For a complicated offshore structure, significant improvement on update rate may be achieved by introducing dynamic geometrical database algorithm.

Secondly, the safety domain size issue. The safety domain is calculated based on the accuracy of the positioning system and orientation sensors. Its size is determined by the performance of the robot positioning system. If a very inaccurate positioning system is used, it may result in the domain size becoming so large that it can not be maneuvered inside an offshore installation. Therefore, the choice robot positioning system will have direct impact on the performance of the system. Our experiments indicate that a 20-centimeter position accuracy with orientation sensor errors less than 5 degrees will make the size of the safety domain about $25 \%$ larger than the real size of the robot. And the size of the safety domain increases as the accuracy degrades. To keep sufficient navigation accuracy, the requirement of positioning accuracy will depend on the complexity of the offshore installation ( e.g. smaller free space will need higher positioning accuracy). Larger size in safety domain inevitably leads to less free space in the workplace the ROV pilot can use to fly the robot. This sometimes may become a crucial issue when navigating the robot in a very confined area within an offshore structure, e.g. approaching a group of complicated structure members.

Finally, the issue of robot positioning accuracy and update rate. Every frame of the virtual image is generated according to the robot's position and orientation data. Therefore, ideally the position and orientation data should be input to the virtual telepresence system in real-time. However, this may be difficult in practice. And update rate of about $10 \mathrm{~Hz}$ might be tolerable when the robot is travelling at low speed. In fact, the speed of an ROV moving inside an offshore installation is controlled at less than 1 knot. In this case, the changes in the virtual environment caused by the transition of the robot between each frame would be not noticeable by human eyes. 
As discussed in the second issue, the accuracy of the robot positioning system will have direct impact on the size of the safety domain. To allow the system to be practically usable for assisting ROV navigation within an offshore installation, a positioning accuracy of better than 50 centimeters and orientation sensor accuracy higher than 5 degrees will be required depending on the size of the free space within the installation.

\section{Conclusions}

This paper addresses the issues concerning underwater robot tele-operation and pilot training by proposing a virtual environment based approach. We have proposed a novel ROV safety domain concept to overcome the robot sensor error problem involved in the virtual environment based underwater teleoperation approach. Evaluation of our prototype system has demonstrated the potentials of applying virtual reality in transforming numerical robot sensor data into 3D visual information representing ROV's spatial location and its surrounding environment. This will overcome the visibility and view-filed constraints imposed by existing camera based telepresence approach. Demo of the pilot training and mission rehearsal features of the prototype system has also received encouraging comments received from offshore operators that virtual environment based approach will provide cost-effective solution for training pilot and enhancing subsea mission planning and execution. However, ROV hydrodynamics should be incorporated into the existing simplified mathematical model of ROV motion so as to generate realistic ROV behaviors in the virtual environment. This will be addressed in our future work.

We have also discussed the issues involved in developing a practical virtual telepresence system and outlined the key areas of further research in this paper. To prove the practical feasibility of using virtual environment based system for ROV tele-operation, we intend to carry out offshore trial after completing the electronic scanning sonar matching software and lab experiment.

\section{Acknowledgments}

We would like to thank the Engineering and Physical Sciences Research Council (EPSRC), UK, and Mobil North Sea Ltd for their funding to the work cited in this paper.

\section{REFERENCES}

[1] Jons O P, Ryan J C, Jones G W, Using Virtual Environments in The Design of Ships, Naval Engineers Journal, May, 1994, pp91-106.

[2] Hattori, K. 1994, Looking at Cyberspace from Japan, Virtual Reality Case Book, Van Nostrand Reinhold, ISBN 0-42-01776-6, pp133-137.

[3] Miner N E; Stansfield S A, "Interactive Virtual Reality Simulation System for Robot Control and Operator Training", Proceedings of IEEE International Conference on Robotics, San Diego, CA , USA, 8-13 May 1994.

[4] Loftin R.B., Kenney P., "Training the Hubble Space Telescope Flight Team," IEEE Computer Graphics \& Applications 15 (5), September, 1995, pp. 31-37. 
[5] Hodges L. F., Kooper R., Meyer T. C., Rothbaum B. O., Opdyke D., Graaff J. J. de., Williford J. S., North M. M.: Virtual Environments for Treating the Fear of Heights. IEEE Computer 28(7), 1995, pp27-34

[6] Tate D L, Sibert L E, King L T, Using Virtual Environments To Train Firefighters, IEEE Computer Graphics and Applications, Vol. 17, No. 6, 1997, pp. 23-29.

[7] Yagel R., Stredney D., Wiet G.J., Schmalbrock P., Rosenberg L., Sessanna D.J., Kurzion Y., Building a virtual environment for endoscopic sinus surgery simulation, Computers \& Graphics (Pergamon) v 20 n 6 Nov-Dec,1996. p 813-823.

[8] Kalawsky, R. S.(1994), "The Science of Virtual Reality and Virtual Environments", Addison-Wesley Publishing Company, Inc., 1994.

[9] Bejczy A K, Virtual reality in robotics, Proceedings of the 1996 IEEE Conference on Emerging Technologies and Factory Automation, USA, 1996, p 7-15.

[10] Fletcher B, Harris S, Development of a virtual environment based training system for ROV pilots, Proceedings of the 1996 MTS/IEEE Oceans, USA, 1996, p 65-71

[11] Kim W S and Bejczy A K, "Demonstration of a High-Fidelity Predictive/Preview Display Technique for Telerobotic Servicing in Space," IEEE Trans. on Robotics and Automation, vol. 9, no. 5, 1993, pp 698-702.

[12] Mclaren N, Sonar Based System for Underwater Navigation, PhD thesis, University of Strathclyde, 1994.

[13] Hallset J O, and Berre G, Modular Integrated Man-machine Interaction and Control, in Proceedings of IEEE OCEANS 94, Brest, France, 1994, pp II-108 to II-112.

[14] Otsuka K, Uchida T, Development and Sea-Trial of Ultra-High Sensitive Underwater Color Video Camera, in Proceedings of Intervention/ ROV'92, San Diego, CA USA, 1992, pp 389-393.

[15] Zheng G, Zheng B, A New Laser 3-D Image Information Display Method for ROV, in Proceedings of Intervention/ ROV'92, San Diego, CA USA, 1992, pp183-188,.

[16] Gordon A and Boston B, Clear Imaging in Muddy Waters, The Journal of Offshore Technology,V1, No.2 August 1993, pp34-38.

[17] Oderud T, Kjennerud E, Man-machine Interactions Applied to ROV Operations, in Proceedings of Intervention/ROV'91, Hollywood, Florida, 1991, pp 37- 41.

[18] Vedeler B, "Subsea Work Tasks, Diving Operations, and the Use of Remote Control Vehicles", Marine Technology, Vol 13, No.2, 1992.

[19] Kamgar-Parsi B, Johnson B, Folds D, Belcher E, High-resolution Underwater Acoustic Imaging with Lens-based Systems, International Journal of Imaging Systems and Technology (Special Issue), vol. 8, 1997, pp. 377-385. 
[20] Belcher E, Folds D, Johnson B, Kamgar-Parsi B, and Scroggins D, "3-D Acoustic Imaging with a Thin Lens," Proceedings of the Oceans '93 Conference, October, 1993.

[21] OmniTech AS, 3D Acoustic Cameras and Imaging, http://www.naval-technology.com/ contractors/mine_disposal/omnitech/index.html, 1997.

[22] Hughes V J and Stobart R K, Advanced Computer Based Mission Planning and Execution Support for ROV Operators, in Proceedings of Intervention/ ROV'90, Vancouver, USA, 1990.

[23] Su S, Lee C and Hsu W, "Automatic Generation of Goal Regions for Assembly Tasks in the Presence of Uncertainty", IEEE Transactions on Robotics and Automation, Vol.12, NO.2. 1996, p.

[24] Guha S, Suri D, Suzuki I, "Random probing to approximate medial axes and plan safe motion", Proceedings of International Conference on Advanced Robotics, USA, 1997, p 353-358.

[25] Curto B, Moreno V, "Mathematical formalism for the fast evaluation of the configuration space", Proceedings of IEEE International Symposium on Computational Intelligence in Robotics \&Automation, 1997, p194-199.

[26] Inui M, Kaneda M, "Configuration space based analysis of position uncertainties of toleranced parts in an assembly", Journal of the Japan Society of Precision Engineering. v63 n8 1997. p 1086-1090.

[27] Rosell J, Basanez L, Suarez R, "Embedding rotations in translational configuration space", Proceedings of IEEE International Conference on Robotics and Automation. USA, 1997, p 28252830.

[28] Hsu D, Latombe J C, Motwani R, "Path planning in expansive configuration spaces", Proceedings of IEEE International Conference on Robotics and Automation. USA, 1997, p 2719-2726.

[29] Choi B K, Kim D H, Jerard R B, "C-space approach to tool-path generation for die and mould machining", Computer-Aided Design. v 29 n 9, 1997. p 657-669.

[30] Kim W S, Schenker P S, Bejczy A K, S Leake, and S Ollendorf, "An Advanced Operator Interface Design with Preview/Predictive Displays for Ground-Controlled Space Telerobotic Servicing," SPIE Conference 2057: Telemanipulator Technology and Space Telerobotics, USA, 1993,pp. 96-107.

[31] Lin, Q, “A Virtual Environment Based System for Assisting Underwater Navigation”, PhD thesis, University of Strathclyde, UK, 1997.

[32] Lin, Q and Kuo C, "Virtual Tele-Operation of Underwater Robots", in Proceedings of 1997 IEEE International Conference on Robotics and Automation, New Mexico, USA, 1997, pp1022-1027.

[33] Lin, Q and Kuo C, "A Virtual Reality Interface for Navigation of Unmanned Underwater Vehicles", in Proceedings of IEA’97, Tampere, Finland, 1997. 
[34] Lin, Q and Kuo C, "Virtual Reality for Subsea Intervention Work", in Proceedings of First International Conference on Offshore Oil and Gas Research and Development, Aberdeen, UK, June 1996.

[35] Kuo C and Lin, Q, "Virtual Reality - An Attractive Opportunity for Subsea Technology", in Proceedings of Subsea Technology Conference, Aberdeen, UK, 1997.

[36] McLaren, N.D., Kuo, C. and Pandelus, A., "A Novel System for Underwater Vehicle Navigation In and Around Offshore Structure”, proceedings of ISOPE'93 Conference, Singapore, 1993.

[37] Kuo C., Mclaren N. and Pandelus A., A Self-contained Navigation System for Use by A UUV operating Around Subsea Installations, Executive Summary Report of TUUV, Marine Technology Directorate, Feb.1995, Aberdeen, UK, pp38-43.

\section{BIOGRAPHIES}

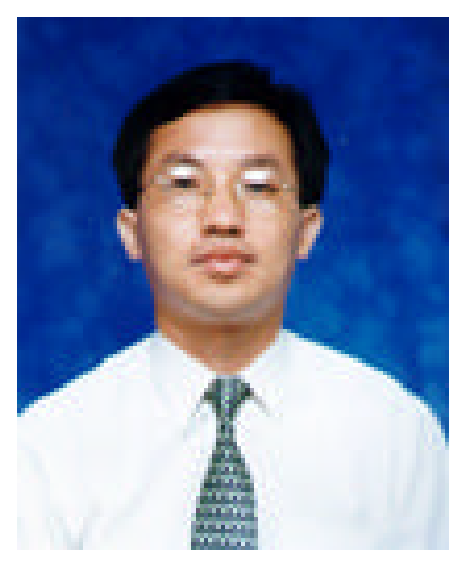

Dr Qingping Lin

Dr Qingping Lin received his $\mathrm{PhD}$ in Computer Applications from University of Strathclyde, UK, in 1997. He is currently an Assistant Professor with Information Communication Institute of Singapore (ICIS), School of Electrical \& Electronic Engineering, Nanyang Technological University. Between 1984 and 1994, he was a faculty member of Jimei University, P.R.China. His current research interests include virtual reality, large-scale collaborative virtual environment (CVE), Object-Oriented software development, and CVE based CASE tool. Dr Lin's research work has led to two international patents filed and one spin-off company (cu3D Technologies Pte Ltd, http://www.cu3d.com/, invested by Incubator@Work, Singapore Technologies Group. Some of his research results are commercialized in the spin-off company for applications in web-based 3D collaborative eLearning, engineering visualization, eManual, on-line exhibition, simulations, and virtual showrooms. Dr Lin is also a member of Editorial Board of International Journal of Information Technology. 
Contact information for Qingping Lin:

Qingping Lin

School of Electrical \& Electronic Engineering Information Communication Institute of Singapore Nanyang Technological University

Nanyang Avenue

SINGAPORE 639798

Telephone: (65) 67904688

Email link: mailto:iqplin@ntu.edu.sg

Contact information for Chengi Kuo:

Professor Chengi Kuo

Department of Ship and Marine Technology

The University of Strathclyde

100 Montrose Street

Glasgow G4 0LZ

Tel. +44 (0) 1415524400

Fax. +44 (0) 1415522879

Email link: mailto:c.kuo@strath.ac.uk

web site: http://www.strath.ac.uk/ 